\title{
Mesenteric ischaemia following posterior myocardial infarction
}

\author{
Authors: Mansoor Zafar, ${ }^{\mathrm{A}}$ Mariya Farooq, ${ }^{\mathrm{B}}$ Ahmed Abousamra, ${ }^{\mathrm{C}}$ Andrew Marshall ${ }^{\mathrm{D}}$ and Mark Whitehead ${ }^{\mathrm{E}}$
}

\begin{abstract}
An 84-year-old man was admitted with urinary tract infection and chest discomfort. He initially responded to conservative acute coronary syndrome management and antibiotics.

On day 6 of admission, he developed acute severe abdominal pain; 12-lead electrocardiography showed widespread ST-segment depression in the anterior chest leads with STelevation in the posterior leads (V7-9) suggestive of an acute posterior myocardial infarction. Arterial blood gases showed severe metabolic acidosis with a lactate of $11 \mathrm{mmol} / \mathrm{L}$.

An urgent computed tomography angiography suggested acute small bowel ischaemia. The case was discussed with the on-call surgical team, who advised that, due to severe frailty, he was not fit for surgical intervention and should be managed conservatively. He was managed with intravenous heparin infusion and supportive measures, but sadly continued to deteriorate and was palliated. He died shortly afterwards.
\end{abstract}

KEYWORDS: mesenteric ischaemia, posterior myocardial infarction

DOI: 10.7861/clinmed.2021-0218

\section{Case presentation}

A frail 84-year-old man presented to the hospital with a 3-day history of feeling feverish and non-specific chest discomfort. He had a known background of recent onset of exertional chest pain suggestive of angina, primary stable Raynaud's disease, stable prostate cancer, severe frailty and chronic kidney disease with estimated glomerular filtration rate (eGFR) of $31 \mathrm{~mL} / \mathrm{min} /$ $1.73 \mathrm{~m}^{2}$. His initial 12-lead electrocardiography (ECG) in the emergency department showed sinus rhythm with incomplete right-bundle branch block (RBBB) morphology and left anterior fascicular block. There was minor T-wave inversion in the septal leads (V1 and V2) felt to be part of the RBBB pattern. Baseline

Authors: ${ }^{\text {A }}$ specialty registrar in general internal medicine and gastroenterology, Conquest Hospital, St Leonards-on-Sea, UK; ${ }^{B}$ core trainee 3 and education fellow, Conquest Hospital, St Leonards-on-Sea, UK; ${ }^{C}$ foundation year 2 doctor, Conquest Hospital, St Leonards-on-Sea, UK; ${ }^{D}$ consultant cardiologist, Conquest Hospital, St Leonards-on-Sea, UK; ${ }^{E}$ consultant physician and gastroenterologist, Conquest Hospital, St Leonards-on-Sea, UK troponin T was raised at 1,052 ng/L, and 6 hours later 2,299 ng/L. Urine dip-stick was positive for nitrite and leukocytes, although subsequent urine culture was negative. As a result, the patient was admitted for cardiac monitoring and received treatment with triple therapy for acute coronary syndrome (ACS) with aspirin, clopidogrel, fondaparinux, intravenous fluids and antibiotics. Initially, he responded well to the initial management and remained pain-free.

On the sixth day of the admission, while awaiting inpatient coronary angiography, he developed severe mid-abdominal pain. On examination there was severe generalised mid-abdominal pain associated with guarding. Subsequently, ECG showed extensive ischaemic changes suggestive of acute posterior myocardial infarction (MI; Fig 1). He was given sublingual glyceryl trinitrate spray which had little to no effect. Pain responded marginally to intravenous $5 \mathrm{mg}$ of morphine. Arterial blood gases (ABGs) showed lactate of $11 \mathrm{mmol} / \mathrm{L}$. He was closely monitored and aggressive fluid resuscitation resulted in effectively lowering the lactate to $7 \mathrm{mmol} / \mathrm{L}$ (Table 1). Urgent bloods with serial troponin levels were $794 \mathrm{ng} / \mathrm{L}$ and 2,356 $\mathrm{ng} / \mathrm{L}$ and eGFR of $21 \mathrm{~mL} / \mathrm{min} / 1.73 \mathrm{~m}^{2}$. He had an urgent computed tomography angiography that showed severe small bowel ischaemia (Fig 2).

In view of the patient's severe frailty, and his rapid deterioration, it was decided to provide best supportive care. He received an intravenous heparin infusion along with subcutaneous morphine on an 'as needed' basis. The family agreed with the poor prognosis and understood the acute deterioration. The patient died peacefully the following morning.

\section{Discussion}

Often the ST-segment changes on ECG accompanied with chest pain are associated with and indicative of cardiac ischaemia. However, there are cases reported with ECG findings of cardiac ischaemia who later developed abdominal pathology.

The peak incidence of bowel ischaemia has been reported to be in people's sixth and seventh decade with almost equal male to female distribution, although, some studies have demonstrated male predominance. ${ }^{1,2}$ The mortality of patients reporting bowel ischaemia remains high at $80 \%{ }^{1}$. This has reported to be due to difficulties in making an early diagnosis, followed by intestinal infarction with sepsis with its sequalae. ${ }^{3}$

A case of a 63-year-old woman has been reported who had border line anterior ST-segment elevation in anterior leads (namely V1 and V2) with complaints of epigastric pain, who later 
Fig 1. a) Electrocardiography showing posterior myocardial infarction with ST segment depression in V1, V2, V3 and V4. b) Posterior lead electrocardiography with V7, V8 and V9 showing ST-segment elevation indicative of posterior myocardial infarction.

a

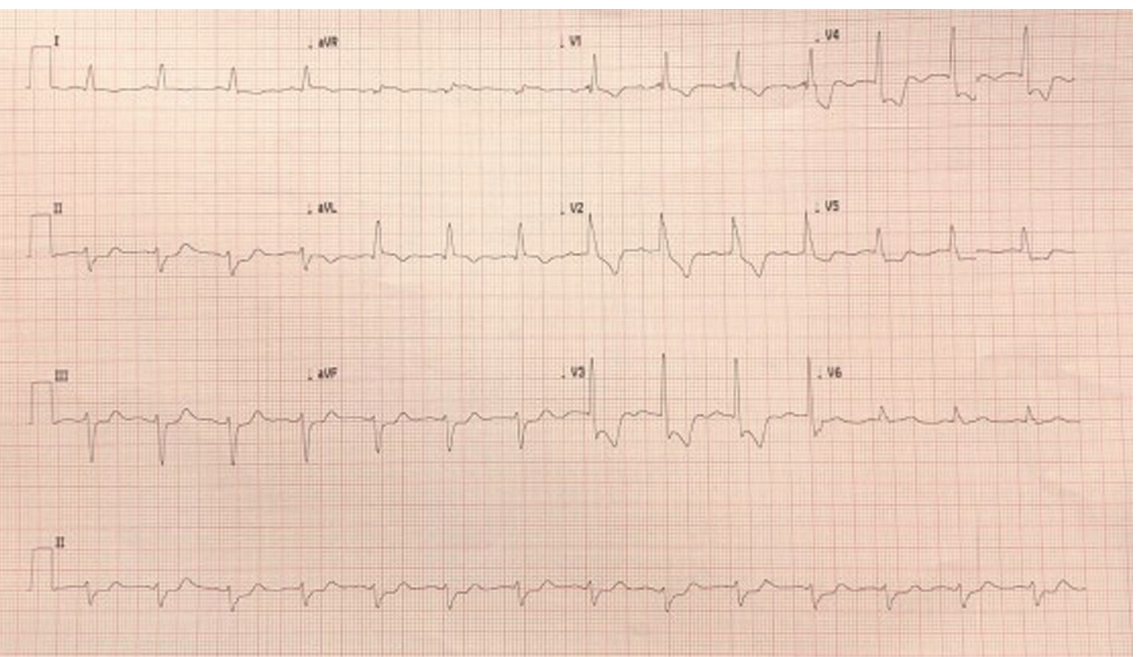

b

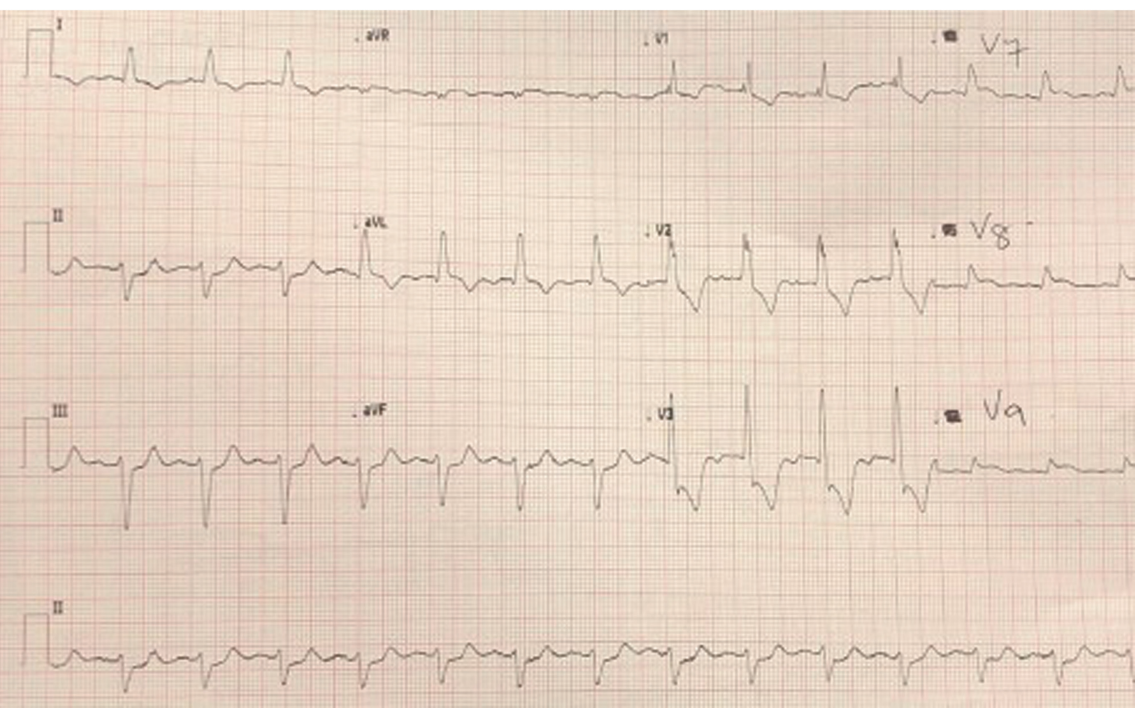

Table 1. Arterial blood gas with increased anion-gap metabolic acidosis with high lactate

$\begin{array}{lll} & \text { Initial } & \begin{array}{l}\text { Following fluid } \\ \text { resuscitation }\end{array} \\ \mathrm{pH} & 7.04 & 7.20 \\ \text { Partial pressure of carbon dioxide, } \mathrm{kPa} & 6 & 4.3 \\ \text { Partial pressure of oxygen, } \mathrm{kPa} & 11 & 16.5 \\ \text { Sodium, } \mathrm{mmol} / \mathrm{L} & 135 & 136 \\ \text { Potassium, } \mathrm{mmol} / \mathrm{L} & 4.1 & 3.8 \\ \text { Chloride, } \mathrm{mmol} / \mathrm{L} & 102 & 102 \\ \text { Calcium, } \mathrm{mmol} / \mathrm{L} & 1.17 & 1.15 \\ \text { Glucose, } \mathrm{mmol} / \mathrm{L} & 6.6 & 8.6 \\ \text { Lactate, } \mathrm{mmol} / \mathrm{L} & 11 & 7 \\ \text { Oxygen concentration, \% } & 96 & 97.7 \\ \text { Oxygen saturation, \% } & 98 & 99.2 \\ \text { Base excess, } \mathrm{mmol} / \mathrm{L} & -18.1 & -14.4 \\ \text { Bicarbonate, } \mathrm{mmol} / \mathrm{L} & 9.9 & 19.4\end{array}$

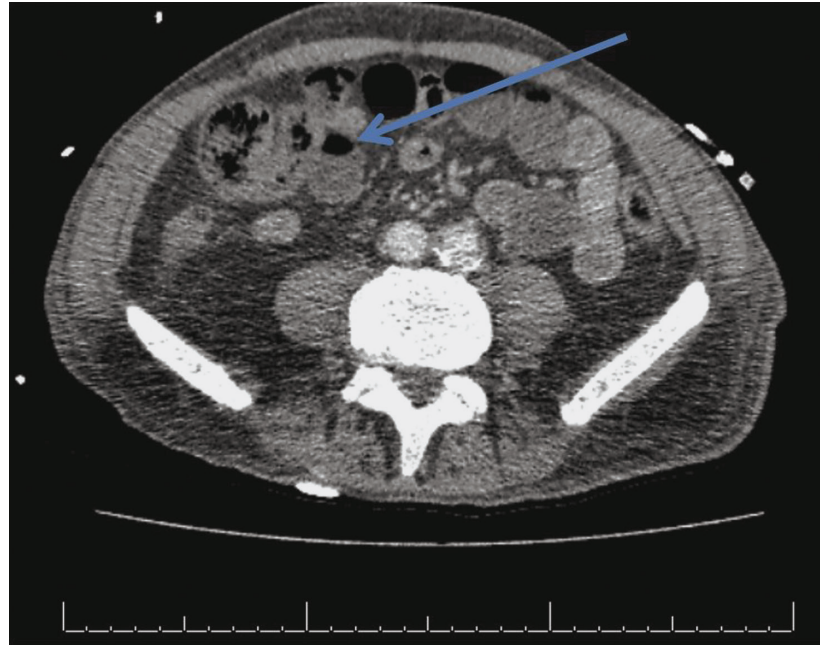

Fig 2. Computed tomography of the abdomen and pelvis. Prominent but not significantly dilated small bowel loops in the pelvis which contains air-fluid level (arrow). Hypoperfusion / lack of enhancement of their wall suggestive of small bowel ischaemia. 
underwent exploratory laparotomy with a removal of ischaemic bowel. ${ }^{4}$

A novel case of inferior MI with rise in troponin levels has been reported for a 61-year-old man, who presented with vomiting and diarrhoea and later had right hemi-colectomy. ${ }^{5}$

Another case of inferior MI with atrial fibrillation with rising troponin levels and increase lactate of $3.1 \mathrm{mmol} / \mathrm{L}$ (normal range $0.7-2.7$ ) has been reported. The patient later underwent exploratory laparotomy with removal of a necrotic caecal portion of the bowel. ${ }^{6}$

A case of a 74-year-old man has been reported among the case series report, with severe bowel ischaemia and rapid deterioration who post-operatively died following massive gastrointestinal haemorrhage. $^{3}$

In the case we describe, the sequence of events may either be an acute MI followed by emboli leading to bowel ischaemia or that the MI caused low cardiac output and then ischaemia as prior mesenteric stenosis presents with bowel ischaemia. Nevertheless, the bowel ischaemia with high lactate of $11 \mathrm{mmol} / \mathrm{L}$ seems to be associated with posterior MI and with poorer outcome in a severe frail patient.

We, to our best knowledge, present the first case of severe bowel ischaemia with ECG findings of acute posterior ST-segment elevation myocardial infarction. Additionally, we noticed a significant rise in lactate levels in our patient following posterior MI. Although there are occasional successful stories reported previously, there remain an increase in inpatient intra-operative and post-operative mortality and morbidity associated with low lactate levels in anterior and inferior MI. It remains questionable if, after excluding severe frailty, whether better intra-operative and post-operative outcome is expected in patients presenting with bowel ischaemia following posterior MI. It will be interesting to see more cases towards these ECG findings and abdominal complaints to help clinicians be aware of this phenomenon during acute medical on calls.

\section{Key points}

> It is important for clinicians to be aware that severe bowel ischaemia may present in the context of acute myocardial infarction, including ECG findings of posterior MI as in our patient. This may be the result of thrombo-embolic events secondary to myocardial infarction with left ventricular mural thrombus or atrial fibrillation, or associated with severely reduced cardiac output.

> Any patient with a complaint of abdominal pain, with a background of ischaemic heart disease, should get arterial blood gases done to look for a rise in lactate. This relatively quick and minimally invasive diagnostic evaluation could prevent the patient from undergoing an invasive heart catheterisation with its substantial attendant sequelae and morbidity burden.

> A prompt computed tomography of the abdomen and pelvis with contrast is vital in early diagnosis of bowel ischaemia.

\section{References}

1 Shaw RS, Rutledge RH. Superior-mesenteric-artery embolectomy in the treatment of massive mesenteric infarction. N Engl J Med 1957;257:595-8.

2 Corder AP, Taylor L. Acute mesenteric ischaemia. Postgrad Med J 1993:69:1-3.

3 Heys SD, Brittenden J, Crofts T]. Acute mesenteric ischaemia: the continuing difficulty in early diagnosis. Postgrad Med J 1993; 69:48-51.

4 Koci F, Eltibi R, Hadley M, Kumar D. Necrotic bowel induces takotsubo-like myocardial injury. Tex Heart Inst J 2014;41:638-40.

5 Yeh YT, Tu CM, Wu YW. Mesenteric ischemia mimicking ST-segment elevation myocardial infarction. N Engl J Med 2015; 373:780-1.

6 Baldeo C, Seegobin K, Yaranov D, Rollini F. Malignant right coronary artery presenting as an st-segment elevation myocardial infarction-a case report. J Geriatr Cardiol 2018;15:467-8.

Address for correspondence: Dr Mansoor Zafar, Conquest Hospital, The Ridge, St Leonards-on-Sea, East Sussex TN37 7RD, UK.

Email:1mansoorzafar@gmail.com 